\title{
Leaky modes in multilayer uniaxial optical waveguides
}

\author{
Lluis Torner, Fernando Canal, and J. Hernandez-Marco
}

\begin{abstract}
The propagation characteristics of the leaky modes in planar anisotropic waveguides with a multilayer structure have been investigated by means of a compact rigorous formalism. The leakage losses and leaky transition angle have been studied for the fundamental and first hybrid modes. An inhomogeneous waveguide and buffered step index type structure have been discussed. Particular attention has been devoted to the variation of the loss coefficient of the leaky modes as a function of buffer thickness and buffer refractive index. A notably different behavior has been obtained for various configurations. Keywords: Leaky modes, waveguide theory, integrated optics.
\end{abstract}

\section{Introduction}

A great number of both active and passive integrated optical devices are fabricated by using uniaxial anisotropic materials, such as $\mathrm{LiNbO}_{3}$ or $\mathrm{LiTaO}_{3}$. Electrooptic modulators, switches, mode converters, couplers, and polarization controllers have been made on titanium-diffused lithium niobate. ${ }^{1,2}$ The detailed knowledge of the properties of the anisotropic optical waveguiding structures appears highly useful to improve the optimization level of such devices, and, also, it may suggest a new class of device based specifically on waveguide anisotropy. ${ }^{3}$

For homogeneous three-layer structures exact analytical solutions are available in the literature. This problem, in addition to the inhomogeneous case, has been analyzed with great detail by several authors, ${ }^{4-12}$ who have pointed out the interesting properties of such waveguides. In particular, leakage losses were theoretically described and experimentally measured..$^{13,14}$ The bidimensional case has also been studied by means of the coupled-mode theory ${ }^{15}$ and the effective index method. ${ }^{16}$ However, because of the rising performances required by the increasingly sophisticated optical circuits, there has been in recent years growing interest in multilayer waveguides. These structures are widely used since they provide more choices to the designer in addition to its particular properties. Concerning the leaky modes in such structures, interest in them comes from their connection with the mode-

\footnotetext{
The authors are with Polytechnic University of Catalonia, Department of Signal Theory \& Communications, Apdo. 30 002, 08080 Barcelona, Spain.

Received 13 October 1988.

0003-6935/90/182805-10\$02.00/0.

(C) 1990 Optical Society of America.
}

dependent loss waveguides and anisotropy based cutoff devices. Nevertheless, although the theory of modal dispersion in multilayer isotropic waveguides has been very well described, its anisotropic counterpart is not yet fully developed.

In this paper we use the transfer-matrix method to establish the waveguiding condition for light propagation in planar uniaxial dielectric waveguides with a multilayer structure with no restrictions on the optical axes orientations. This method provides a very compact formalism to analyze such structures, and it has been extensively used in the isotropic case. ${ }^{17}$ It is based on the well known $4 \times 4$ formalism, which has been developed by Berreman ${ }^{18}$ and Vassell ${ }^{19}$ and in a different way by Yeh. ${ }^{20}$ A new formulation was reported recently by Knoesen et $a .^{21}$ and for special cases by Walpita. ${ }^{22}$ The approach can be summarized as follows. In the homogeneous substrate and superstrate the total fields are a superposition of the ordinary and extraordinary waves propagating in a uniaxial unbounded medium. These solutions are continued across the intermediate dielectric medium by means of a characteristic matrix containing the field solutions. Thus the required boundary conditions on the top and bottom waveguide interfaces are expressed by a determinantal condition which yields to the waveguiding condition. The procedure can be applied to the exact analytical study of uniaxial multilayer structures and the numerical analysis of inhomogeneous uniaxial waveguides.

Details of the analysis are given in Sec. II. As an application of the formalism, in Sec. III we investigated the leaky-mode propagation in both an inhomogeneous waveguide and a step index structure with a dielectric buffer layer. The leakage losses of the waveguide have been studied for the fundamental and first hybrid modes for different optical axis orientations. In the first case, we mainly devoted our attention to the 
effects introduced by the inhomogeneity. In the second case, the study was focused to the attenuation coefficient of the leaky modes and the guided-to-leaky mode transition angle as a function of the buffer thickness and buffer refractive index.

\section{Transfer-Matrix Approach}

An asymmetric configuration of uniaxial crystals forming a three-layer structure with arbitrarily oriented optical axes is assumed (Fig. 1). In the principal axis coordinate system of the crystals, the dielectric tensor takes the form

$$
\left[\epsilon_{D}=\left(\begin{array}{ccc}
\epsilon_{o} & & \\
& \epsilon_{o} & \\
& & \epsilon_{e}
\end{array}\right)\right. \text {, }
$$

$\epsilon_{o}$ and $\epsilon_{e}$ being the ordinary and extraordinary permittivities, respectively. For a given orientation of the optical axis $(\hat{c})$ determined by the polar and azimuthal angles $(\theta, \varphi)$, the components of the dielectric tensor are obtained from Eq. (1) by means of the application of the associated rotation transformation $\mathcal{R}(\theta, \varphi)$. Thus one obtains

$$
\begin{aligned}
& \epsilon_{x x}=\epsilon_{o}\left(\sin ^{2} \varphi+\cos ^{2} \varphi \cos ^{2} \theta\right)+\epsilon_{e} \cos ^{2} \varphi \sin ^{2} \theta, \\
& \epsilon_{y y}=\epsilon_{o}\left(\cos ^{2} \varphi+\sin ^{2} \varphi \cos ^{2} \theta\right)+\epsilon_{e} \sin ^{2} \varphi \sin ^{2} \theta, \\
& \epsilon_{z z}=\epsilon_{o} \sin ^{2} \theta+\epsilon_{e} \cos ^{2} \theta, \\
& \epsilon_{x y}=\left(\epsilon_{e}-\epsilon_{o}\right) \sin \varphi \cos \varphi \sin ^{2} \theta, \\
& \epsilon_{x z}=\left(\epsilon_{e}-\epsilon_{o}\right) \cos \varphi \sin \theta \cos \theta, \\
& \epsilon_{y z}=\left(\epsilon_{e}-\epsilon_{o}\right) \sin \varphi \sin \theta \cos \theta .
\end{aligned}
$$

In a waveguide such as the one described above only hybrid modes can propagate except for specially symmetric $\hat{c}$-axis orientations. If the propagation direction is taken to be along the $\hat{x}$-axis and assuming time harmonic dependence, the fields at any point have the form $\exp [i(\beta x-\omega t)], \beta$ being the propagation constant. Outside the guiding layer the fields must be evanescent so that

$$
\begin{aligned}
& \mathbf{E}_{\mathrm{c}}(z)=\mathbf{E}_{\mathrm{c}} \exp \left(\gamma_{\mathrm{c}} z\right), z<0, \\
& \mathbf{E}_{s}(z)=\mathbf{E}_{s} \exp \left[\gamma_{s}(D-z)\right], z>D,
\end{aligned}
$$

where the subscripts $c$ and $s$ stand for superstrate and substrate regions, respectively. By substitution of Eqs. (3) and (4) into the wave equation,

$$
\nabla^{2} \mathbf{E}+\mu \omega^{2}[\epsilon] \mathbf{E}=\nabla(\nabla \cdot \mathbf{E}),
$$

and taking into account Eq. (2), an homogeneous equation system is obtained, the determinant of which must vanish. This condition leads to two possible solutions for the decay constants $\gamma_{c, s}$ that correspond to the ordinary and extraordinary waves

$$
\begin{aligned}
& \gamma_{o}= \pm \sqrt{\beta^{2}-\mu \omega^{2} \epsilon_{o}}, \\
& \gamma_{e}= \pm \frac{1}{\epsilon_{z z}} \sqrt{\beta^{2}\left(\epsilon_{x x} \epsilon_{z z}-\epsilon_{x z}^{2}\right)-\mu \omega^{2} \epsilon_{o} \epsilon_{e} \epsilon_{z z}}( \pm) i \frac{\epsilon_{x z}}{\epsilon_{z z}} \beta .
\end{aligned}
$$

Here the upper sign in the parentheses holds for the

\section{Superstrate $[\epsilon]_{c}$}

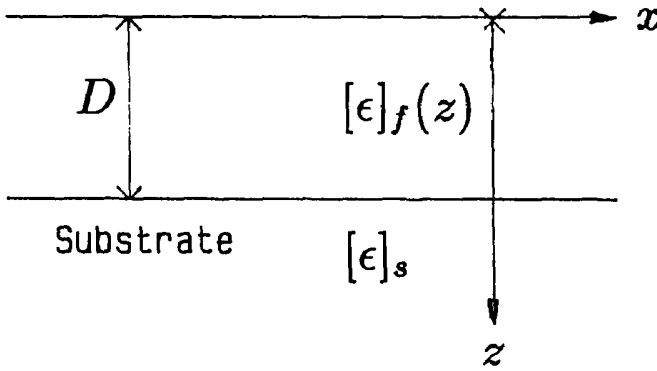

Fig. 1. Schematic waveguiding multilayer structure. Propagation is along $x$.

substrate and the lower for the superstrate. According to the transfer-matrix method, at any pair of transversal planes $z_{1}, z_{2}$ the tangential components of the fields can be related by using a matrix $T$, which may be defined as

$$
\left[\begin{array}{l}
E_{x} \\
H_{y} \\
E_{y} \\
H_{x}
\end{array}\right]_{z_{2}}=T\left[\begin{array}{l}
E_{x} \\
H_{y} \\
E_{y} \\
H_{x}
\end{array}\right]_{z_{1}}
$$

The existence of this transfer matrix is guaranteed by the linearity of the Maxwell equations. In fact, the matrix $T$ contains the field solutions in the region between the planes $z_{1}, z_{2}$, so that in Eq. (8) it continues the solution from $z_{1}$ to $z_{2}$ across the intermediate region. Let us take $z_{1}=0$ and $z_{2}=D$. Thus the required boundary conditions for the tangential field components are necessarily verified if the field solution is (8) join with the known solutions (3)-(4) in the substrate and cover. This condition can be written in a matrix form using the following standard procedure. The existence of solutions (6) and (7) requires that all the components of the fields be expressed in terms of two of them. Then we have chosen as independent variables the $\hat{y}$ and $\hat{x}$ components of the electric field associated with the ordinary and extraordinary waves, respectively. In this way, the electric field in the superstrate $(z<0)$ can be written as

$$
\mathbf{E}_{c}(z)=\left[\begin{array}{c}
\Lambda_{x o} \\
1 \\
\Lambda_{z o}
\end{array}\right] E_{y o} \exp \left(\gamma_{o} z\right)+\left[\begin{array}{c}
1 \\
\Lambda_{y e} \\
\Lambda_{z e}
\end{array}\right] E_{x e} \exp \left(\gamma_{e} z\right)
$$

and in a more compact notation

$$
\mathbf{E}_{c}(z)=\left[\begin{array}{cc}
\Lambda_{x o} & 1 \\
1 & \Lambda_{y e} \\
\Lambda_{z o} & \Lambda_{z e}
\end{array}\right]\left\{\begin{array}{l}
\left.E_{y o} \exp \left(\gamma_{o} z\right)\right\} \\
E_{x e} \exp \left(\gamma_{e} z\right)
\end{array}\right\}
$$

For the magnetic field one has

$$
\mathbf{H}_{c}(z)=\frac{-i}{\mu \omega}\left[\begin{array}{cc}
-\gamma_{o} & -\gamma_{e} \Lambda_{y e} \\
\gamma_{0} \Lambda_{x o}-i \beta \Lambda_{z o} & \gamma_{e}-i \beta \Lambda_{z e} \\
i \beta & i \beta \Lambda_{y e}
\end{array}\right]\left\{\begin{array}{l}
E_{y o} \exp \left(\gamma_{o} z\right) \\
E_{x e} \exp \left(\gamma_{e} z\right)
\end{array}\right\} .
$$

The coefficients $\Lambda$ appearing in these expressions are obtained from Eq. (5). Again in $z<0$ one has 


$$
\begin{aligned}
& \Lambda_{x o}=\frac{\epsilon_{x y} \gamma_{o}^{2}-i \epsilon_{y z} \gamma_{o} \beta}{\left(\epsilon_{o}-\epsilon_{x x}\right) \gamma_{o}^{2}+\beta^{2}\left(\epsilon_{o}-\epsilon_{z z}\right)+2 i \epsilon_{x z} \gamma_{o} \beta}, \\
& \Lambda_{z o}=\frac{\epsilon_{x y}\left(\mu \omega^{2} \epsilon_{x z}-i \gamma_{o} \beta\right)-\epsilon_{y z}\left(\gamma_{o}^{2}+\mu \omega^{2} \epsilon_{x x}\right)}{\left(\epsilon_{o}-\epsilon_{x x}\right) \gamma_{o}^{2}+\beta^{2}\left(\epsilon_{o}-\epsilon_{z z}\right)+2 i \epsilon_{x z} \gamma_{o} \beta}, \\
& \Lambda_{y e}=\mu \omega^{2} \frac{\epsilon_{x y}\left(\beta^{2}-\mu \omega^{2} \epsilon_{z z}\right)+\epsilon_{y z}\left(\mu \omega^{2} \epsilon_{x z}-i \gamma_{e} \beta\right)}{\left(\gamma_{e}^{2}-\beta^{2}+\mu \omega^{2} \epsilon_{y y}\right)\left(\mu \omega^{2} \epsilon_{z z}-\beta^{2}\right)-\left(\mu \omega^{2} \epsilon_{y z}\right)^{2}}, \\
& \Lambda_{z e}=\frac{\left(\gamma_{e}^{2}-\beta^{2}+\mu \omega^{2} \epsilon_{y y}\right)\left(i \gamma_{e} \beta-\mu \omega^{2} \epsilon_{x z}\right)+\left(\mu \omega^{2}\right)^{2} \epsilon_{x y} \epsilon_{y z}}{\left(\gamma_{e}^{2}-\beta^{2}+\mu \omega^{2} \epsilon_{y y}\right)\left(\mu \omega^{2} \epsilon_{z z}-\beta^{2}\right)-\left(\mu \omega^{2} \epsilon_{y z}\right)^{2}} .
\end{aligned}
$$

On the other hand, according to Eq. (4), the corresponding expressions for the substrate write in the same way as Eqs. (10)-(15) by making the transformation $\gamma \rightarrow-\gamma$ for both the ordinary and extraordinary waves. Thus one has

$$
\begin{aligned}
& \mathbf{E}_{s}(z)= {\left[\begin{array}{cc}
\Lambda_{x o}^{*} & 1 \\
1 & \Lambda_{y e}^{*} \\
\Lambda_{z o}^{*} & \Lambda_{z e}^{*}
\end{array}\right]\left\{\begin{array}{l}
\left.E_{y o}^{*} \exp \left[\gamma_{o}^{*}(D-z)\right]\right\} \\
E_{x e}^{*} \exp \left[\gamma_{e}^{*}(D-z)\right]
\end{array}\right\}, } \\
& \mathbf{H}_{s}(z)=\frac{-i}{\mu \omega}\left[\begin{array}{cc}
\gamma_{o}^{*} & \gamma_{e}^{*} \Lambda_{y e}^{*} \\
-\left(\gamma_{o}^{*} \Lambda_{x o}^{*}+i \beta \Lambda_{z o}^{*}\right) & -\left(\gamma_{e}^{*}+i \beta \Lambda_{z e}^{*}\right) \\
i \beta & i \beta \Lambda_{y e}^{*}
\end{array}\right] \\
& \times\left\{\begin{array}{l}
E_{y o}^{*} \exp \left[\gamma_{o}^{*}(D-z)\right] \\
E_{x e}^{*} \exp \left[\gamma_{e}^{*}(D-z)\right]
\end{array}\right\} .
\end{aligned}
$$

Throughout the superscript * indicates a substrate parameter. Substituting Eqs. (10), (11) and (16), (17) into Eq. (8) an homogeneous equation system is obtained which can be written as

$$
\left[\begin{array}{cccc}
\Lambda_{x o}^{*} & 1 & \nu_{1 o} & \nu_{1 e} \\
\tau_{o}^{*} & \tau_{e}^{*} & \nu_{2 o} & \nu_{2 e} \\
1 & \Lambda_{y e}^{*} & \nu_{3 o} & \nu_{3 e} \\
-\gamma_{o}^{*} & -\gamma_{e}^{*} \Lambda_{y e}^{*} & \nu_{4 o} & \nu_{4 e}
\end{array}\right]\left(\begin{array}{l}
E_{y o}^{*} \\
E_{x e}^{*} \\
E_{y o} \\
E_{x e}
\end{array}\right)=0,
$$

where

$$
\begin{aligned}
& \tau_{o}^{*} \equiv \gamma_{o}^{*} \Lambda_{x o}^{*}+i \beta \Lambda_{z o}^{*}, \\
& \tau_{e}^{*} \equiv \gamma_{e}^{*}+i \beta \Lambda_{z e}^{*}, \\
& \nu_{j} \equiv\left\{\begin{array}{cc}
-\Omega_{j}, & j=1,3, \\
i \mu \omega \Omega_{j}, & j=2,4,
\end{array}\right.
\end{aligned}
$$

with

$$
\begin{aligned}
& \Omega_{j o} \equiv T_{j 1} \Lambda_{x o}+T_{j 3}-\frac{i}{\mu \omega}\left(T_{j 2} \tau_{o}-\gamma_{o} T_{j 4}\right), \\
& \Omega_{j e} \equiv T_{j 1}+T_{j 3} \Lambda_{y e}-\frac{i}{\mu \omega}\left(T_{j 2} \tau_{e}-\gamma_{e} \Lambda_{y e} T_{j 4}\right) .
\end{aligned}
$$

As is explicit in these expressions, the $\nu_{j}$ functions must be evaluated in the superstrate. The condition for having a nontrivial solution in Eq. (18) is that the determinant of the coefficients vanishes. This condition leads to a restriction of the possible values of the propagation constant $\beta$, which must now form a discrete set, and, therefore, it is the waveguiding condition. After straightforward manipulation it can be expressed as

$$
\begin{aligned}
& \Lambda_{x o}^{*}\left[\tau_{e}^{*} N_{34}-\Lambda_{y e}^{*} N_{24}-\gamma_{e}^{*} \Lambda_{y e}^{*} N_{23}\right]-\tau_{o}^{*}\left[N_{34}-\Lambda_{y e}^{*} N_{14}-\gamma_{e}^{*} \Lambda_{y e}^{*} N_{13}\right] \\
& +\left[N_{24}-\tau_{e}^{*} N_{14}-\gamma_{e}^{*} \Lambda_{y e}^{*} N_{12}\right]+\gamma_{o}^{*}\left[N_{23}-\tau_{e}^{*} N_{13}+\Lambda_{y e}^{*} N_{12}\right]=0,
\end{aligned}
$$

where

$$
N_{j l} \equiv \nu_{j o} \nu_{l e}-v_{j e} \nu_{l o} .
$$

Equation (24) has been obtained for arbitrary waveguide parameters with no restriction on the optical axis orientation of the crystals. Then it is noticeable that for the orientations which allow separable TE-TM field solutions Eq. (24) is notably simplified. This situation happens when one (or both) substrate and superstrate is (or are) isotropic (i.e., $\epsilon_{o}=\epsilon_{e}$ ) and also when the optical axis lies in the same plane as the propagation direction and is contained in the plane perpendicular to that of the waveguide (in our notation $\varphi=0^{\circ}$ ). As well, when $\varphi=\theta=90^{\circ}$, the field solutions allow a TE-TM decomposition. In the second case, when the $\hat{c}$-axis lies in the $\varphi=0^{\circ}$ plane in both the substrate and superstrate, the expressions for the $\mathbf{E}$ and $\mathbf{H}$ fields are identical to Eqs. (10), (11), (16), and (17), but now $\Lambda_{x o}=0, \Lambda_{y e}=0, \Lambda_{z o}=0$, and

$$
\Lambda_{z e}=\frac{\mu \omega^{2} \epsilon_{x z}-i \gamma_{e} \beta}{\beta^{2}-\mu \omega^{2} \epsilon_{z z}}
$$

The waveguiding condition is obtained as for the general case. Thus it can be written in a formally identical way to the isotropic structure ${ }^{19}$ in terms of the new variables:

$$
\begin{aligned}
& \tilde{\epsilon} \equiv \frac{\epsilon_{o} \epsilon_{e}}{\epsilon_{z z}}, \\
& \tilde{\beta} \equiv \beta \sqrt{\frac{\tilde{\epsilon}}{\epsilon_{z z}}}, \\
& \tilde{\gamma}_{e} \equiv \sqrt{\tilde{\beta}^{2}-\mu \omega^{2} \tilde{\epsilon}}=\gamma_{e}+i \frac{\epsilon_{x z}}{\epsilon_{z z}} \beta,
\end{aligned}
$$

and similar expressions for $\tilde{\epsilon}^{*}, \tilde{\beta}^{*}$, and $\tilde{\gamma}_{e}^{*}$ (Appendix A). It is well known that ${ }^{5,6,19,21}$ if the optical axis of the uniaxial media forming the guiding layer lies also in the $\varphi=0^{\circ}$ plane, the waveguide supports pure TE and TM modes. As usual, in this case Eq. (24) splits into two (one TE and one TM) very simple expressions. Concerning the $\varphi=\theta=90^{\circ}$ case, it can be considered as well by taking the corresponding expressions for the isotropic case but with $\epsilon=\epsilon_{o}$ in the TM terms and $\epsilon=\epsilon_{e}$ in the TE ones. Likewise in a great number of practical applications the superstrate is isotropic and the substrate is a uniaxial crystal. In this case one also has $\Lambda_{x o}=0, \Lambda_{y e}=0, \Lambda_{z o}=0$, and $\Lambda_{z e}=-i \beta / \gamma_{c}$, where

$$
\gamma_{\mathrm{c}}= \pm \sqrt{\beta^{2}-k_{0}^{2} n_{c}^{2}}
$$

$n_{c}$ and $k_{0}$ being the refractive index of the isotropic medium and the free-space wavenumber, respectively. Then the waveguiding condition is given by Eq. (24), but now Eqs. (22) and (23) simplify to

$$
\Omega_{j o}=T_{j 3}+\frac{i \gamma_{c}}{\mu \omega} T_{j 4}
$$




$$
\Omega_{j e}=T_{j 1}+\frac{i k_{0}^{2} n_{c}^{2}}{\mu \omega \gamma_{c}} T_{j 2}
$$

The transfer matrix of an homogeneous uniaxial dielectric slab can be analytically calculated by using the $4 \times 4$ formalism. In the general case, it contains a superposition of the two ordinary and two extraordinary waves propagating in a birefringent medium. In the case of multilayer step index waveguides, the transfer matrix of the structure is obtained by the product of all the matrices associated with each sublayer, in accordance with the ordering criterion prescribed in Eq. (8). When diffused waveguides are considered, in which the inhomogeneity takes place in addition to the anisotropy, the problem becomes more complicated. In such a case a closed-form solution of Maxwell equations is not generally available, and the associated transfer matrix must be evaluated with the help of approximate methods or numerical techniques. The latter can be made through the direct integration of Maxwell equations in the diffusion region by means of such standard numerical procedures as the RungeKutta method ${ }^{11}$ or the Gear predictor technique, ${ }^{16}$ which are available in most mathematical libraries. Also, the well known multilayer staircase technique can be useful in analyzing especially complex structures containing homogeneous additional layers. In this case the inhomogeneous region is considered as a finite set of thin homogeneous films, for each the transfer matrix is analytically known.

Finally, since we have imposed no restrictions on the existence of complex values of the propagation constant $\beta$, the above derived expressions are applicable to both guided and leaky modes. Thus it is worth noticing that the square roots appearing in Eqs. (6) and (7) for the transverse propagation constant at the substrate and superstrate $\left(\gamma_{o}, \gamma_{e}\right)$ must be evaluated with the right sign consistent with the behavior of mode fields far from the waveguide. Special attention is required when considering leaky modes due to their improper nature. ${ }^{10,12,23}$

\section{Discussion}

As an application of the formalism developed in the preceding section, we have analyzed here the propagation characteristics of the leaky modes of various multilayer waveguides fabricated in dielectic uniaxial materials. Such modes come from the frustration on total internal reflection at the interfaces between the guiding layer and surrounding dielectric media and are obtained as complex solutions of the eigenvalue equation. Concerning the anisotropic case, with a suitable orientation of the crystal optical axes, one of the polarizations (ordinary and extraordinary) suffers leakage losses, whereas the other remains guided in the film. These kinds of mode are leaky guided modes in contrast to the leaky unguided modes which occur in the isotropic case. ${ }^{24}$

We have considered here the case in which the optical axes of the crystals lie in the waveguide plane $(\theta=$ $90^{\circ}$ ), making an angle $\varphi$ with the positive $\hat{x}$-axis with the same value in all uniaxial media. The eigenvalue equation associated with this case is obtained from $\mathrm{Eq}$. (24) by taking into account the suitable characteristic matrix of the structure. The explicit expression of this characteristic matrix is given in Appendix B. A waveguide such as the one described above can support only hybrid modes with the six field components. When the optical axis makes a small angle with the waveguide axis $\left(\varphi \approx 0^{\circ}\right)$ the field components associated with the ordinary and extraordinary waves are weakly coupled and the modes correspond to the TETM polarizations. When the angle increases, the coupling between the above components grows also and several modes become leaky. The nature of these leaky modes is described in Refs. 7-10. Here we discuss some open questions from which new results come.

\section{A. Inhomogeneous Waveguide}

First, we have analyzed an asymmetric inhomogeneous waveguide with a Gaussian profile in both the ordinary and extraordinary refractive indices, and in which the cover is in air. The various waveguide parameters are

$$
G_{1}:\left\{\begin{array}{cc}
n_{o f}=2.2946 & n_{e f}=2.2108 \\
n_{o s}=2.2866 & n_{e s}=2.2028 \\
n_{\mathrm{c}}=1 & D=2 \mu \mathrm{m}
\end{array}\right\} .
$$

Here $D$ is the characteristic depth of the Gaussian profile, and the subscript $f$ stand for film parameters. Also we assumed $\lambda=633 \mathrm{~nm}$. When $\varphi=0^{\circ}, G_{1}$ supports the $T E_{0}$ and $T M_{0}$ modes. The mode which is the $\mathrm{TE}_{0}$ mode at $\varphi=0^{\circ}$ remains guided for all values of $\varphi$ and becomes the $\mathrm{TM}_{0}$ mode at $\varphi=90^{\circ}$ via a predominantly ordinary hybrid mode. On the other hand, the mode that at $\varphi=0^{\circ}$ is $\mathrm{TM}_{0}$ becomes leaky beyond $\varphi_{l} \simeq$ $11^{\circ}$, and it converts into the $\mathrm{TE}_{0}$ at $\varphi=90^{\circ}$ by means of a predominantly extraordinary hybrid mode. These modes are referred to as the $\left[\mathrm{TE}_{0}, \mathrm{TM}_{0}\right][g]$ and $\left[\mathrm{TM}_{0}, \mathrm{TE}_{0}\right][l]$, respectively. Here $[\mathrm{g}]$ and $[l]$ indicate a guided and leaky mode. We deal now with the behavior of the loss coefficient of the $\left[\mathrm{TM}_{0}, \mathrm{TE}_{0}\right][l]$ mode.

In Fig. 2 we have plotted the loss coefficient of the above mentioned leaky mode as a function of the angle $\varphi$. To perform the calculations use has been made of a numerical zoom root-finding algorithm to solve directly the eigenvalue equation (24) for complex roots. Although this procedure requires a considerable number of iterations in the complex $\beta$-plane, it provides very accurate results. The graded index profile has been introduced in the formalism through the multilayer staircase technique. First, the accurate numerical results obtained for a few values of the angle $\varphi$ are given in Table I together with the results reported by Koshiba et al. ${ }^{12}$ from the finite element technique. As can be seen, the agreement between both sets of values is quite good, the differences being unsignificant in practice.

On the other hand, our main aim in this case is to examine the effects of inhomogeneity on the loss coefficient. Thereupon, we included also in Fig. 2 the 


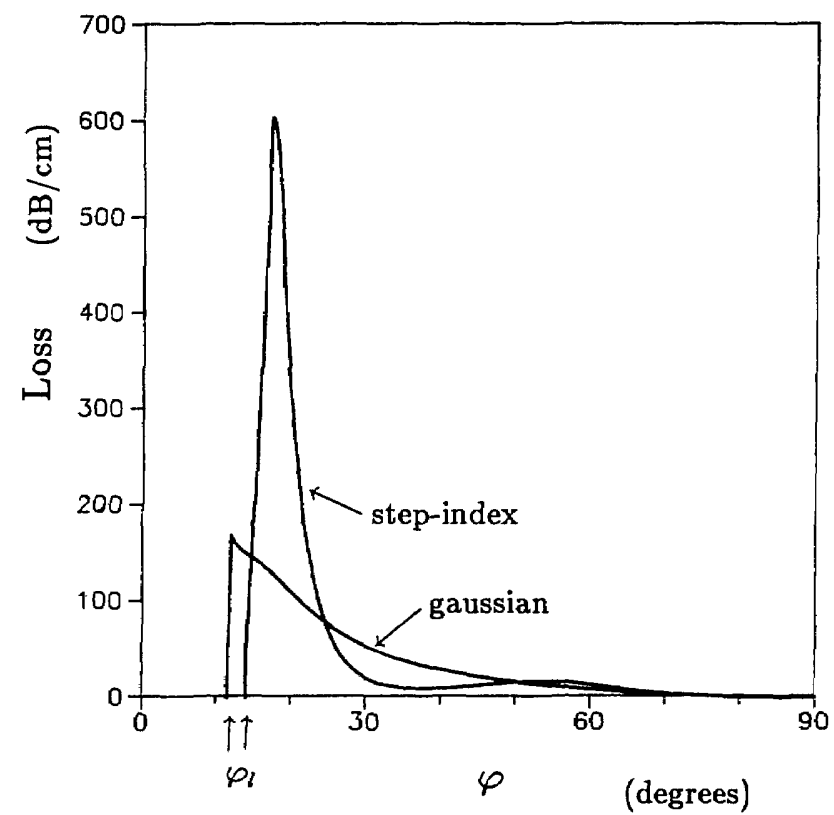

Fig. 2. Loss coefficient of the $\left[\mathrm{TM}_{0}, \mathrm{TE}_{0}\right][l]$ mode supported by $G_{1}$ as a function of the optical axis orientation. $\varphi_{l}$ is the guided-toleaky mode transition angle.

Table I. Accurate Numerlcal Values Obtained for the Waveguide with a Gausslan Profile $\left(G_{1}\right)$ and Comparison with the Finite Element Results Reported In fief. 12

\begin{tabular}{|c|c|c|c|c|}
\hline \multirow{2}{*}{$\varphi$} & \multicolumn{2}{|c|}{ Transfer Matrix } & \multicolumn{2}{c|}{ Finite Element } \\
\cline { 2 - 5 } & $\Re e\left(\beta / k_{0}\right)$ & Loss $(\mathrm{dB} / \mathrm{cm})$ & $\Re e\left(\beta / k_{0}\right)$ & Loss $(\mathrm{dB} / \mathrm{cm})$ \\
\hline $12^{\circ}$ & 2.28576 & 170.1 & 2.28574 & 181.6 \\
\hline $30^{\circ}$ & 2.26757 & 54.0 & 2.26756 & 53.8 \\
\hline $60^{\circ}$ & 2.22573 & 8.9 & 2.22572 & 8.9 \\
\hline
\end{tabular}

results corresponding to a waveguide identical to $G_{1}$ but with a step index profile. This plot deserves some comments. First, in the case of the homogeneous profile the loss coefficient shows a secondary maximum which is not observed for the Gaussian profile (see also Fig. 3). Also, the step index waveguide shows a pronounced loss peak which does not appear in the inhomogeneous case. These features agree with the previsions of Burns et al. ${ }^{8}$ in the sense that the sharp structure showed by the loss coefficient in a step index waveguide is due to interferential phenomena which originate in the abrupt discontinuities at the filmcover and film-substrate interfaces. The same conclusions follow from Figs. 4 and 5 , where the loss coefficient of the $\left[\mathrm{TM}_{0}, \mathrm{TE}_{0}\right][l]$ mode has been plotted as a function of the cover refractive index for two different values of $\varphi$. In all cases, to emphasize the effects due to the Gaussian profile the charts have been referred to the value of the loss coefficient for $n_{c}=1$. First, it can

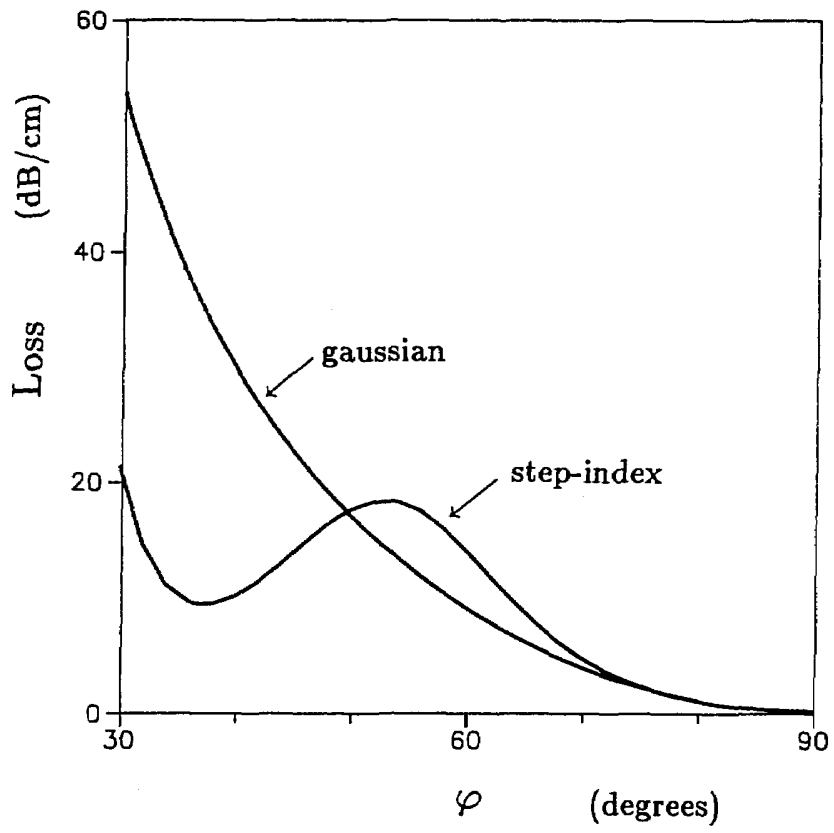

Fig. 3. Detail of Fig. 2 showing the secondary maximum of the loss coefficient as a function of $\varphi$ for the homogeneous waveguide and the monotonous decrease which occurs for the Gaussian profile.

be seen in both figures that the loss coefficient depends on $n_{c}$ in a stronger way for the Gaussian profile than for the step index one. Also, in the case of the homogeneous waveguide, the behavior of the loss coefficient on $n_{c}$ is completely different for $\varphi=30^{\circ}$ than for $\varphi=40^{\circ}$. This difference, which does not occur for the Gaussian profile, comes again from interferential phenomena.

Finally, it is interesting to note that the presence of the graded index profile modifies the value of the critical angle at which the $\left[\mathrm{TM}_{0}, \mathrm{TE}_{0}\right][l]$ mode becomes leaky. As mentioned above $\varphi_{l} \simeq 11^{\circ}$ for the Gaussian profile, whereas $\varphi_{l} \simeq 14^{\circ}$ for the homogeneous waveguide.

\section{B. Multilayer Step Index Structures}

We deal now with multilayer uniaxial structures. The interest in such waveguides comes from their analogy with their isotropic counterparts, whose noticeable properties have been pointed out in several works. Our main motivation on this subject lays in the fact that the presence of additional layers modifies not only the real part of the effective index $\left[\operatorname{Re}\left(\beta / k_{0}\right)\right]$ but also the loss coefficient of the leaky modes and the guidedto-leaky mode transition angle.

In highly asymmetric structures the propagation characteristics of the leaky modes are only slightly modified by the presence of additional layers at the top of the waveguide. This is because in these waveguides the leaky modes mainly account for radiation to the substrate, so that the cover material only affects the leaky modes when its refractive index amounts to a value close to that of the substrate. The situation changes completely when structures with a high degree of symmetry are considered. Now the leaky modes 


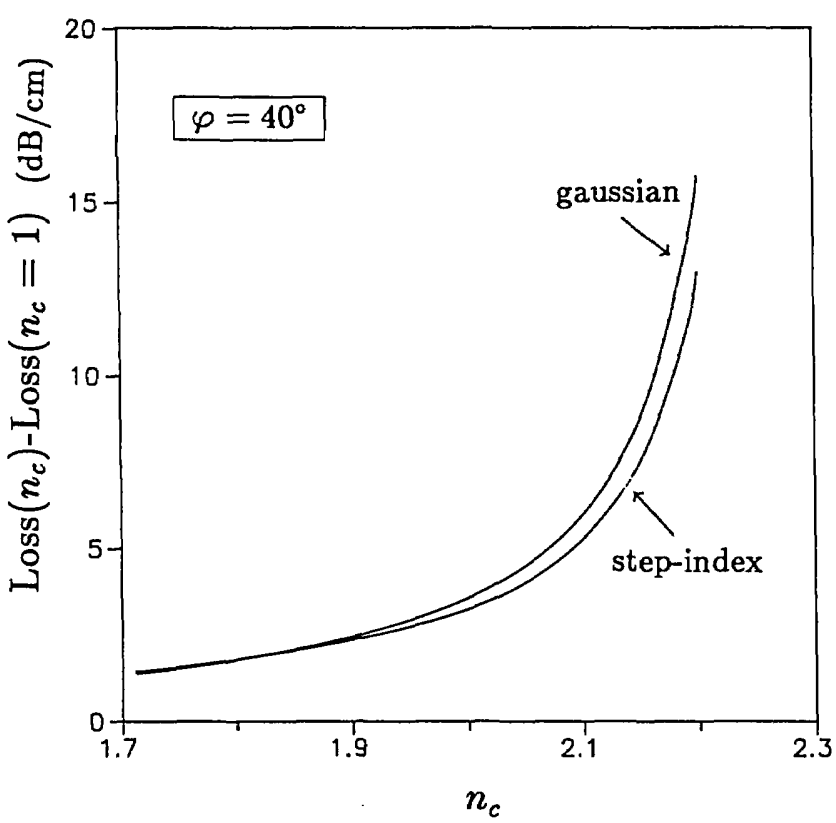

Fig. 4. Loss coefficient for the leaky mode in Fig. 2 as a function of the cover refractive index for $\varphi=40^{\circ}$. To emphasize the effects due to the Gaussian profile the curves have been referred to the value of the loss coefficient for $n_{c}=1$.

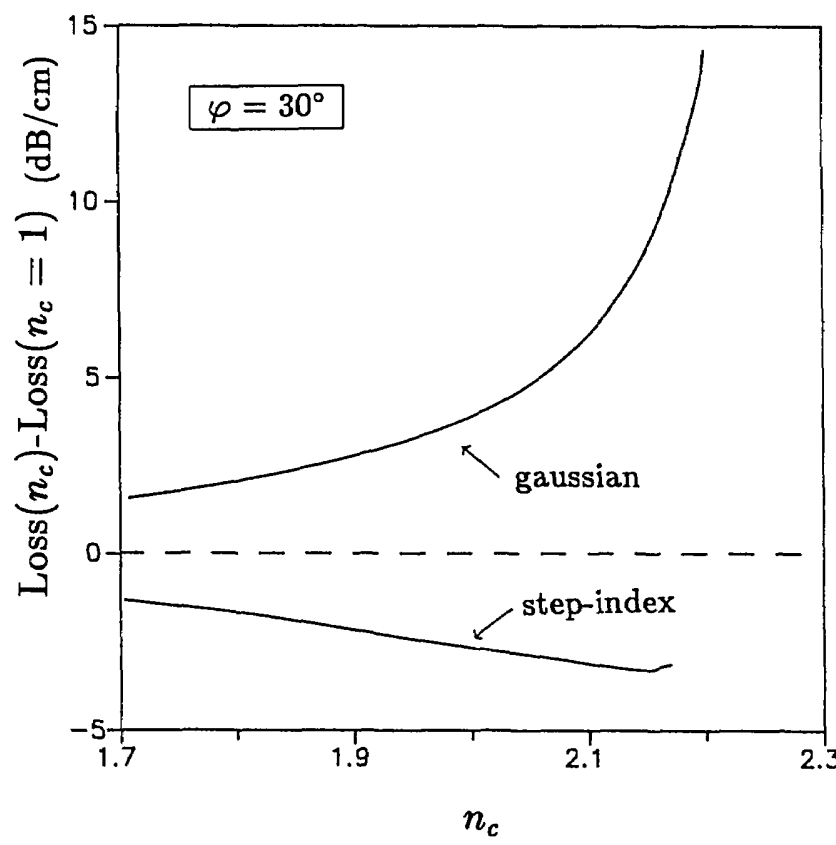

Fig. 5. Same as in Fig. 4 but for $\varphi=30^{\circ}$.

correspond to radiation toward both substrate and superstrate, so that they are strongly dependent on cover parameters. ${ }^{12}$ For example, this is the behavior shown in Figs. 4 and 5, which we have discussed previously.

The formalism developed in Sec. II allows us to analyze in an exact and simple way the multilayer uniaxial waveguides. As already mentioned, the char-

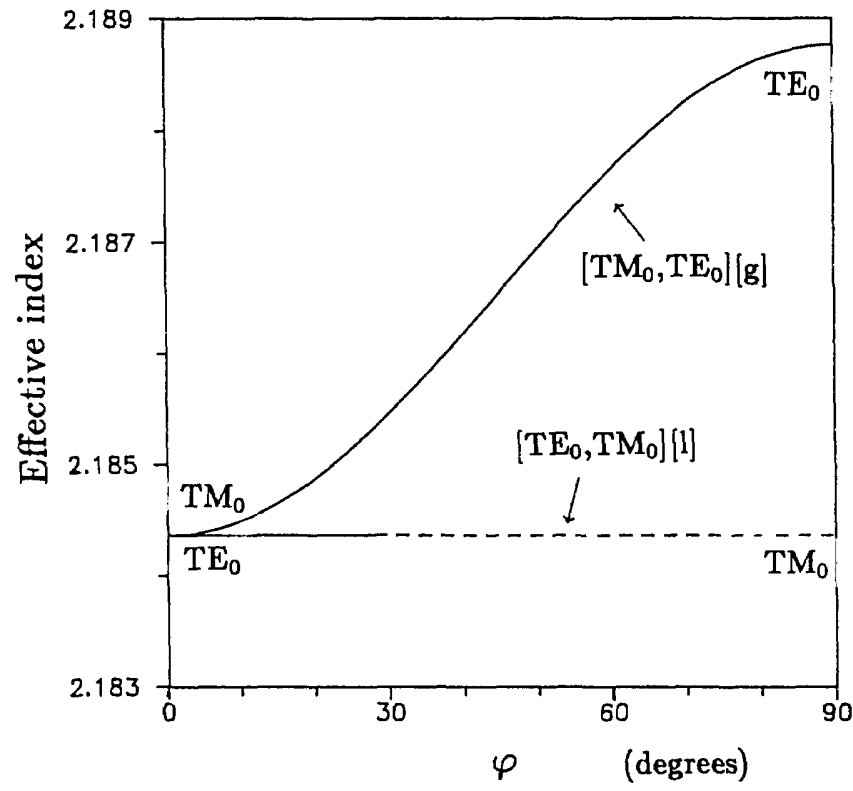

Fig. 6. Effective indices of the hybrid modes supported by $G_{2}$ as a function of $\varphi$. At $\varphi=0,90^{\circ}$ the modes are pure TE and TM. The $\left[\mathrm{TM}_{0}, \mathrm{TE}_{0}\right][g]$ mode is a predominantly extraordinary guided hybrid mode, whereas the $\left[\mathrm{TE}_{0}, \mathrm{TM}_{0}\right][l]$ mode is a predominantly ordinary leaky hybrid mode. Solid line: pure guided mode. Dashed line: leaky mode.

acteristic matrix of the whole structure is obtained by the ordered product of the matrices of each layer. As an example, we have analyzed a step index symmetric waveguide in which cover, substrate, and film are assumed to be uniaxial media. In addition, there is an isotropic buffer layer of thickness $D_{b}$ and refractive index $n_{b}$ placed between the cover and film. Opposite the former case, we consider now a $\mathrm{LiTaO}_{3}$ based waveguide with the following parameters:

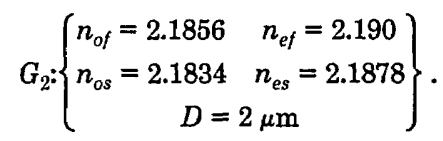

In the limiting cases $\varphi=0^{\circ}$ and $\varphi=90^{\circ}$ this waveguide supports the $\mathrm{TE}_{0}$ and $\mathrm{TM}_{0}$ modes. For any other value of the angle $\varphi, G_{2}$ supports two hybrid modes, which will be denoted as $\left[\mathrm{TM}_{0}, \mathrm{TE}_{0}\right][g]$ and $\left[\mathrm{TE}_{0}, \mathrm{TM}_{0}\right][l]$ according to the notation introduced above. The guided-to-leaky mode transition angle for the leaky mode amounts to $\varphi \simeq 28^{\circ}$. The rolls of the TE and TM modes have been interchanged in relation to the former case $\left(G_{1}\right)$ because now we are dealing with a positive birefringent material $\left(n_{e}>n_{0}\right)$. Figure 6 shows the propagation characteristics of the above modes as a function of $\varphi$.

The influence of the buffer layer on the $\left[\mathrm{TE}_{0}, \mathrm{TM}_{0}\right][l]$ mode has been analyzed in Figs. 7-11. In Fig. 7 we plotted the loss coefficient of this mode as a function of the buffer thickness, and the variation of the loss coefficient with the buffer refractive index is shown in Fig. 8. Two different orientations of the crystal optical axis have been considered: $\varphi=30^{\circ}$ and 


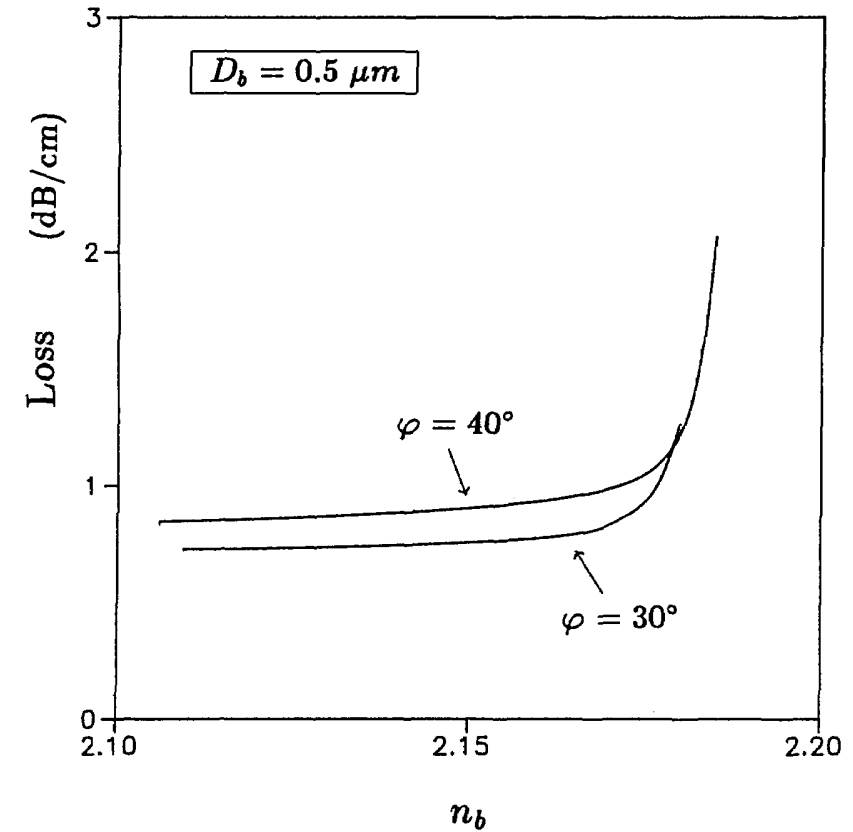

Fig. 7. Loss coefficient as a function of the buffer refractive index. Mode $\left[\mathrm{TE}_{0}, \mathrm{TM}_{0}\right][l]$. Buffer thickness: $D_{b}=0.5 \mu \mathrm{m}$.

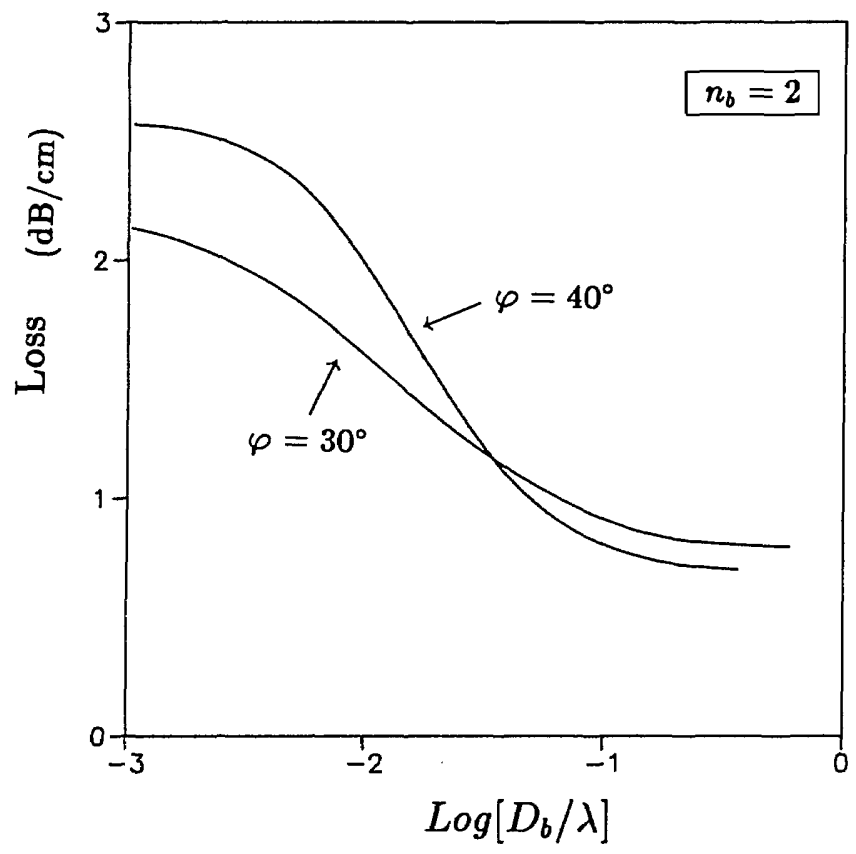

Fig. 8. Loss coefficient as a function of the decimal logarithm of the $\lambda$-scaled buffer thickness. Mode $\left[\mathrm{TE}_{0}, \mathrm{TM}_{0}\right][l]$. Buffer refractive index: $n_{b}=2$.

$\varphi=40^{\circ}$. The charts in these figures show that the effects of the additional layer on the loss coefficient of the leaky mode depends strongly on the buffer thickness and is practically insensitive to the buffer refractive index. In this case, the obtained behavior for the loss coefficient is similar for both crystal orientations. However, this result cannot be generalized. For example, in Figs. 9 and 10 the previous dependences have

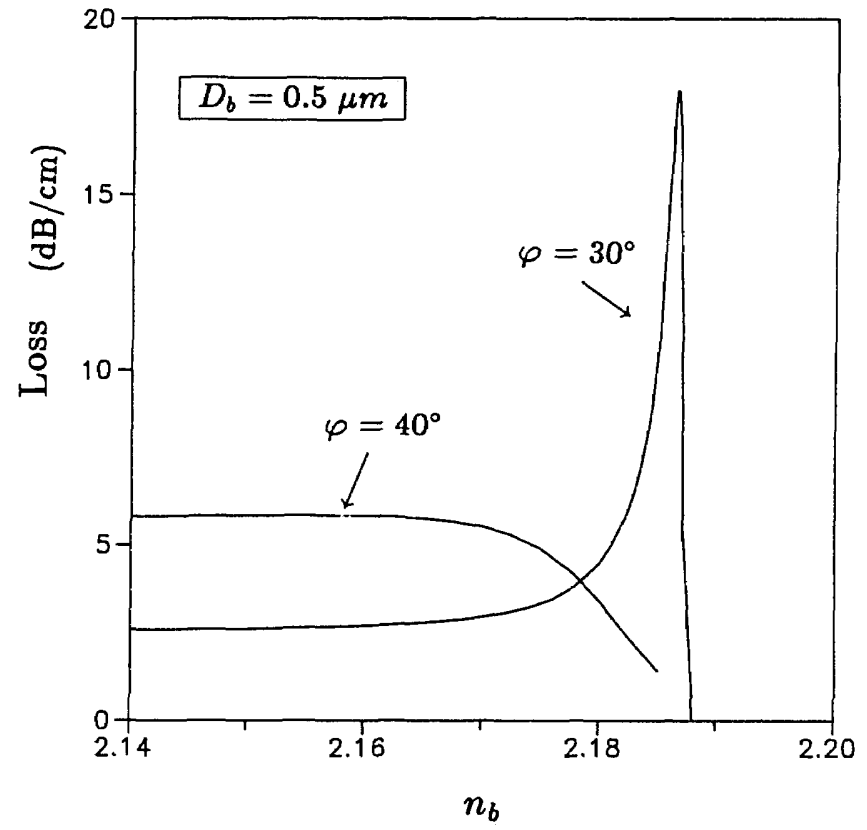

Fig. 9. Same as in Fig. 7 for the $\left[\mathrm{TE}_{1}, \mathrm{TM}_{1}\right][l]$ mode supported by the multimode version $(D=6 \mu \mathrm{m})$ of $G_{2}$.

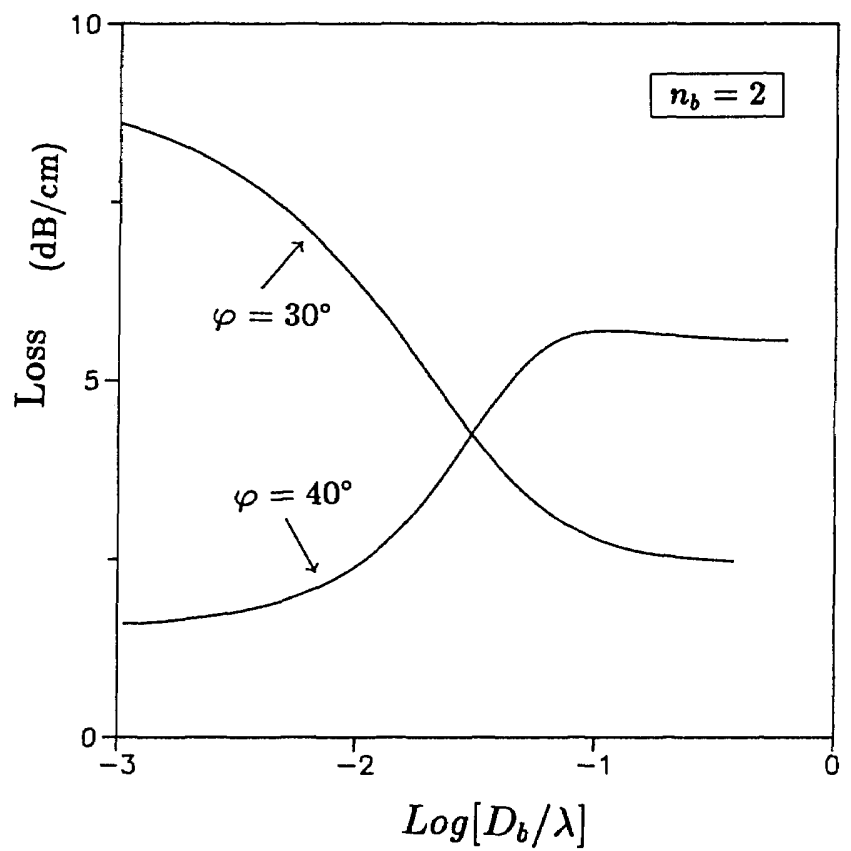

Fig. 10. Same as in Fig. 8 for the $\left[\mathrm{TE}_{1}, \mathrm{TM}_{1}\right][l]$ mode supported by the multimode version $(D=6 \mu \mathrm{m})$ of $G_{2}$.

been plotted for the $\left[\mathrm{TE}_{1}, \mathrm{TM}_{1}\right][l]$ mode supported by a multimode version $(D=6 \mu \mathrm{m})$ of $G_{2}$. In fact, this multimode waveguide also supports the $\left[\mathrm{TM}_{1}, \mathrm{TE}_{1}\right][l]$ mode, but it is a very low loss leaky mode. ${ }^{7}$ As can be seen in Figs. 9 and 10, now the effects due to the buffer layer are completely different for the two considered crystal orientations.

Finally, it is to be emphasized that the presence of 


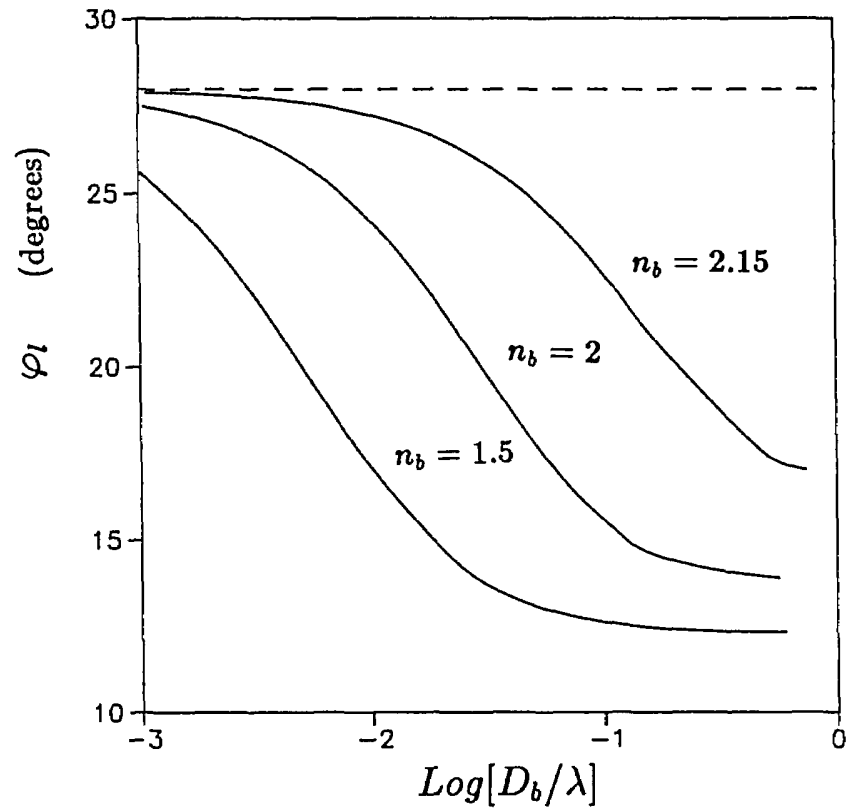

Fig. 11. Guided-to-leaky mode transition angle for the $\left[\mathrm{TE}_{0}, \mathrm{TM}_{0}\right][l]$ mode supported by $G_{2}$ as a function of the decimal logarithm of the $\lambda$-scaled buffer thickness. Dashed line: transition angle in the absence of the buffer layer.

the dielectric low index layer also modifies the value of the guided-to-leaky mode transition angle. For example, in Fig. 11 we plotted the variation of the leaky transition angle with the buffer thickness for various values of the buffer refractive index for the $\left[\mathrm{TE}_{0}, \mathrm{TM}_{0}\right][l]$ mode supported by $G_{2}$. Similar behavior has been obtained for other leaky modes. As in Figs. 7 and 9, the leaky transition angle becomes insensitive to the buffer thickness when $D_{b}$ amounts to a value of the order of $\lambda$. The modification of the guided-to-leaky mode transition angle wtih the waveguide parameters should be particularly interesting for the anisotropy based cutoff devices in which this angle plays a fundamental role. We return to this question in a forthcoming paper. ${ }^{25}$

\section{Concluding Remarks}

The waveguiding condition for light propagation in planar dielectric uniaxial waveguides with a multilayer structure has been obtained with no restrictions on the optical axes orientation. The procedure is based on the extension of the transfer-matrix formalism to include an anisotropic substrate and superstrate. As an application of the formalism we have investigated the propagation characteristics of leaky modes in an inhomogeneous waveguide and a multilayer step index structure in which the crystal optical axes lie in the waveguide plane. The explicit expression of the required characteristic matrix is also given. Both the loss coefficient and the guided-to-leaky mode transition angle have been analyzed as a function of the inhomogeneity and buffer layer parameters. We have shown that the presence of additional low index layers forming multilayer structures with a high degree of symmetry strongly affects the propagation characteristics of the leaky modes supported by such waveguides. These effects depend also on the optical axis orientation due to interferential phenomena which come from the presence of abrupt discontinuities on the interfaces between the various dielectric media.

The authors are grateful to Jiri Ctyroky of the Czechoslovak Academy of Sciences, Prague, for valuable suggestions. This work was partially supported by a grant of the CYCyT of the Spanish Government (PB-87-0798-C0302).

\section{Appendix A}

The waveguiding condition for the case when the $\hat{c}$ axis lies in the plane $\varphi=0^{\circ}$ in both the substrate and superstrate is

$$
\begin{aligned}
& \left\{\mathrm{T}_{21}+i \frac{\omega \tilde{\epsilon}}{\tilde{\gamma}_{e}} \mathrm{~T}_{22}+i \frac{\omega \tilde{\epsilon}^{*}}{\tilde{\gamma}_{e}^{*}} \mathrm{~T}_{11}-\omega^{2} \frac{\tilde{\tilde{\epsilon}} \tilde{\epsilon}^{*}}{\tilde{\gamma}_{e} \tilde{\gamma}_{e}^{*}} \mathrm{~T}_{12}\right\} \times \\
& \left\{\mathrm{T}_{43}+i \frac{\gamma_{o}}{\mu \omega} \mathrm{T}_{44}+i \frac{\gamma_{o}^{*}}{\mu \omega} \mathrm{T}_{33}-\frac{\gamma_{o} \gamma_{o}^{*}}{(\mu \omega)^{2}} \mathrm{~T}_{34}\right\}- \\
& \left\{\mathrm{T}_{23}+i \frac{\gamma_{o}}{\mu \omega} \mathrm{T}_{24}+i \frac{\omega \tilde{\epsilon}^{*}}{\tilde{\gamma}_{e}^{*}} \mathrm{~T}_{13}-\frac{\tilde{\epsilon}^{*} \gamma_{o}}{\mu \tilde{\gamma}_{e}^{*}} \mathrm{~T}_{14}\right\} \times \\
& \left\{\mathrm{T}_{41}+i \frac{\omega \tilde{\epsilon}}{\tilde{\gamma}_{e}} \mathrm{~T}_{42}+i \frac{\gamma_{o}^{*}}{\mu \omega} \mathrm{T}_{31}-\frac{\tilde{\epsilon} \gamma_{o}^{*}}{\mu \tilde{\gamma}_{e}} \mathrm{~T}_{32}\right\}=0 .
\end{aligned}
$$

When the waveguide supports pure TE and TM modes, the first bracket in the above expression corresponds to the TM polarization and the second to the TE one. In such a case, also, the other two brackets vanish identically.

\section{Appendix B}

The transfer matrix of the buffered uniaxial waveguide in Sec. III is given by $\mathrm{T}=\mathrm{U}_{a} \mathrm{U}_{b}$, where $\mathrm{U}_{a}$ and $\mathrm{U}_{b}$ are the matrices associated with the anisotropic film and buffer layer, respectively. Both matrices can be analytically calculated by means of the $4 \times 4$ formalism. The derivation follows a general procedure developed by Vassell, ${ }^{19}$ who has also reported the expression of the transfer matrix for the simpler case of a uniaxial dielectric film whose optical axis lies in the $\varphi$ $=0^{\circ}$ plane. The transfer matrix for the general case can be easily calculated also by means of the same procedure. ${ }^{26}$ In our case, $\theta=90^{\circ}$, it can be written as

$$
\mathrm{U}_{l m}=\left(\lambda_{o}^{2} \alpha_{1}^{2}+\mu \epsilon_{o}\right) \overline{\mathrm{U}}_{l m},
$$

$\overline{\mathrm{U}}$ being an auxiliary matrix whose elements are given by 


$$
\begin{aligned}
& \overline{\mathrm{U}}_{11}=\mu \epsilon_{o} \cos \left(\omega D \lambda_{o}\right)+\lambda_{o}^{2} \alpha_{1}^{2} \cos \left(\omega D \lambda_{e}\right), \\
& \overline{\mathrm{U}}_{12}=i \frac{\lambda_{o}}{\epsilon_{o}}\left[\mu \epsilon_{o} \sin \left(\omega D \lambda_{o}\right)+\frac{\lambda_{o}^{3}}{\lambda_{e}} \alpha_{1}^{2} \sin \left(\omega D \lambda_{e}\right)\right], \\
& \overline{\mathrm{U}}_{13}=\alpha_{1} \lambda_{o}^{2}\left[\cos \left(\omega D \lambda_{o}\right)-\cos \left(\omega D \lambda_{e}\right)\right], \\
& \overline{\mathrm{U}}_{14}=-i \mu \alpha_{1} \lambda_{o}\left[\sin \left(\omega D \lambda_{o}\right)-\frac{\lambda_{o}}{\lambda_{e}} \sin \left(\omega D \lambda_{e}\right)\right], \\
& \overline{\mathrm{U}}_{21}=i \frac{\epsilon_{o}}{\lambda_{o}}\left[\mu \epsilon_{o} \sin \left(\omega D \lambda_{o}\right)+\alpha_{1}^{2} \lambda_{o} \lambda_{e} \sin \left(\omega D \lambda_{e}\right)\right], \\
& \overline{\mathrm{U}}_{23}=i \epsilon_{o} \alpha_{1} \lambda_{o}\left[\sin \left(\omega D \lambda_{o}\right)-\frac{\lambda_{e}}{\lambda_{o}} \sin \left(\omega D \lambda_{e}\right)\right], \\
& \overline{\mathrm{U}}_{24}=-\mu \epsilon_{o} \alpha_{1}\left[\cos \left(\omega D \lambda_{o}\right)-\cos \left(\omega D \lambda_{e}\right)\right], \\
& \overline{\mathrm{U}}_{33}=\alpha_{1}^{2} \lambda_{o}^{2} \cos \left(\omega D \lambda_{o}\right)+\mu \epsilon_{o} \cos \left(\omega D \lambda_{e}\right), \\
& \overline{\mathrm{U}}_{34}=-i \frac{\mu}{\lambda_{e}}\left[\lambda_{o} \lambda_{e} \alpha_{1}^{2} \sin \left(\omega D \lambda_{o}\right)+\mu \epsilon_{o} \sin \left(\omega D \lambda_{e}\right)\right], \\
& \overline{\mathrm{U}}_{43}=-i \frac{\lambda_{o}}{\mu}\left[\lambda_{o}^{2} \alpha_{1}^{2} \sin \left(\omega D \lambda_{o}\right)+\frac{\lambda_{e}}{\lambda_{o}} \mu \epsilon_{o} \sin \left(\omega D \lambda_{e}\right)\right] \\
& \left.\overline{\mathrm{U}}_{31}=-\overline{\mathrm{U}}_{11} \quad \overline{\mathrm{U}}_{44}=\overline{\mathrm{U}}_{33}, \quad \overline{\mathrm{U}}_{32}=-\overline{\mathrm{U}}_{14},\right\} \\
& \left.\overline{\mathrm{U}}_{41}=-\overline{\mathrm{U}}_{23} \quad \overline{\mathrm{U}}_{42}=-\overline{\mathrm{U}}_{13},\right\}
\end{aligned}
$$

where

$$
\begin{aligned}
& \lambda_{o}=\sqrt{\mu \epsilon_{o}-\alpha^{2}}, \\
& \lambda_{e}=\sqrt{\mu \epsilon_{e}-\alpha^{2} \epsilon_{x x} / \epsilon_{o}}, \\
& \alpha_{1}=\frac{\epsilon_{o}-\epsilon_{x x}}{\epsilon_{x y}} .
\end{aligned}
$$

Here $\alpha=\beta / \omega$ and $D$ is the thickness of the anisotropic film. The transfer matrix of the isotropic buffer layer comes directly from the above expressions by making the substitution $\epsilon_{o}=\epsilon_{e}=\varepsilon n_{b}^{2}, \varepsilon$ being the free space permittivity and $n_{b}$ the buffer refractive index. One arrives at the well known expression

$$
\mathrm{U}_{b}=\left(\begin{array}{cc}
u_{\mathrm{TM}} & 0 \\
0 & u_{\mathrm{TE}}
\end{array}\right)
$$

where

$$
u=\left[\begin{array}{cc}
\cos \left(k_{0} D_{b} \beta_{b}\right) & i(\eta / a) \sin \left(k_{0} D_{b} \beta_{b}\right) \\
i(a / \eta) \sin \left(k_{0} D_{b} \beta_{b}\right) & \cos \left(k_{0} D_{b} \beta_{b}\right)
\end{array}\right] .
$$

Here $\eta \equiv \sqrt{\varepsilon / \mu}$ and $D_{b}$ stands for the buffer thickness. Also, use has been made of the definition

$$
\beta_{b} \equiv \sqrt{n_{b}^{2}-N^{2}}
$$

$N \equiv \beta / k_{0}$ being the effective index and $a_{\mathrm{TE}}=-\beta_{b}, a_{\mathrm{TM}}=$ $n_{b}^{2} / \beta_{b}$

\section{References}

1. L. Thylen, "Integrated Optics in $\mathrm{LiNbO}_{3}$ : Recent Developments in Devices for Telecommunications," IEEE/OSA J. Lightwave Technol. LT-6, 847-861 (1988).

2. E. Voges and A. Neyer, "Integrated-Optic Devices on $\mathrm{LiNbO}_{3}$ for Optical Communication," IEEE/OSA J. Lightwave Technol. LT-5, 1229-1238 (1987).

3. T. K. Gaylord and A. Knoesen, "Passive Integrated Optical Anisotropy-Based Devices," J. Mod. Opt. 35, 925-946 (1988).

4. D. P. Gia Russo and J. H. Harris, "Wave Propagation in Anisotropic Thin-Film Optical Waveguides," J. Opt. Soc. Am. 63, 138-145 (1973).

5. W. K. Burns and J. Warner, "Mode Dispersion in Uniaxial Optical Waveguides,” J. Opt. Soc. Am. 64, 441-446 (1974).

6. D. Marcuse, "Modes of a Symmetric Slab Optical Waveguide in Birefringent Media. Part I: Optical Axis not in Plane of Slab," IEEE J. Quantum Electron. QE-14, 736-741 (1978).

7. D. Marcuse and I. P. Kaminow, "Modes of a Symmetric Slab Optical Waveguide in Birefringent Media. Part II: Slab with Coplanar Optical Axis," IEEE J. Quantum Electron. QE-15, 92101 (1979).

8. W. K. Burns, S. K. Sheem, and A. F. Milton, "Approximate Calculation of Leaky-Mode Loss Coefficients for Ti-Diffused $\mathrm{LiNbO}_{3}$ Waveguides," IEEE J. Quantum Electron. QE-15, 1282-1289 (1979).

9. J. Ctyroky and M. Cada, "Generalized WKB Method for the Analysis of Light Propagation in Inhomogeneous Anisotropic Optical Waveguides," IEEE J. Quantum Electron. QE-17, 1064-1070 (1981).

10. J. Ctyroky and M. Cada, "Guided and Semileaky Modes in Anisotropic Waveguides of the $\mathrm{LiNbO}_{3}$ Type," Opt. Commun. 27, 353-357 (1978).

11. E. A. Kolovsky, D. V. Petrov, A. V. Tsarev, and I. B. Yakovkin, "An Exact Method for Analysing Light Propagation in Anisotropic Inhomogeneous Optical Waveguides," Opt. Commun. 43, 21-25 (1982).

12. M. Koshiba, H. Kumagami, and M. Suzuki, "Finite-Element Solution of Planar Arbitrarily Anisotropic Diffused Optical Waveguides," IEEE/OSA J. Lightwave Technol. LT-3, 773-778 (1985).

13. K. Yamanouchi, T. Kamiya, and K. Shibayama, "New Leaky Surface Waves in Anisotropic Metal-Diffused Optical Waveguides," IEEE Trans. Microwave Theory Tech. MTT-26, 298-304 (1978).

14. S. K. Sheem, W. K. Burns, and A. F. Milton, "Leaky-Mode Propagation in Ti-Diffused $\mathrm{LiNbO}_{3}$ and $\mathrm{LiTaO}_{3}$ Waveguides," Opt. Lett. 3, 76-78 (1978).

15. R. A. Stienberg and T. G. Giallorenzi, "Modal Fields of Anisotropic Channel Waveguides," J. Opt. Soc. Am. 67, 523-533 (1977).

16. M. N. Armenise and M. De Sario, "Optical Rectangular Waveguide in Titanium-Diffused Lithium Niobate Having its Optical Axis in the Transverse Plane," J. Opt. Soc. Am. 72, 1514-1521 (1982).

17. J. T. Chilwell and I. J. Hodgkinson, "Thin-Films Field-Transfer Matrix Theory of Planar Multilayer Waveguides and Reflection from Prism-Loaded Waveguides," J. Opt. Soc. Am. A 1, 742-753 (1984).

18. D. W. Berreman, "Optics in Stratified and Anisotropic Media: $4 \times 4$-Matrix Formulation," J. Opt. Soc. Am. 62, 502-510 (1972).

19. M. O. Vassell, "Structure of Optical Guided Modes in Planar Multilayers of Optically Anisotropic Materials," J. Opt. Soc. Am. 64, 166-173 (1974).

20. P. Yeh, "Electromagnetic Propagation in Birefringent Layered Media,” J. Opt. Soc. Am. 69, 742-756 (1979). 
21. A. Knoesen, T. K. Gaylord, and M. G. Moharam, "Hybrid Guided Modes in Uniaxial Dielectric Planar Waveguides," IEEE/ OSA J. Lightwave Technol. LT-6, 1083-1104 (1988).

22. L. M. Walpita, "Solutions for Planar Optical Waveguide Equations by Selecting Zero Elements in a Characteristic Matrix," J. Opt. Soc. Am. A 2, 595-602 (1985).

23. T. Tamir, "Leaky-Wave Antennas," in Antenna Theory, Part 2, R. E. Collin and F. J. Zucker, Eds. (McGraw-Hill, New York, 1969).
24. T. Tamir and F. Y. Kou, "Varieties of Leaky Waves and Their Excitation Along Multilayered Structures," IEEE J. Quantum Electron. QE-22, 544-551 (1986) and references therein.

25. L. Torner and F. Canal, "Guided-to-Leaky Mode Transition in Planar Dielectric Uniaxial Waveguides," submitted for publication.

26. L. Torner, Ph.D. Thesis, U. Politécnica de Catalunya, Barcelona (1989). 